\title{
A Novel Method for Calculating Approximate Weight of an Object through its Image
}

\author{
${ }^{1}$ Amaresh Chandra Meher, ${ }^{2}$ Uddgam Singh and ${ }^{3}$ K.K. Senapati, \\ ${ }^{1}$ Dept of Computer Science, SUIT, Jyoti Vihar, Sambalpur-768019, India \\ 2,3 Dept of Computer Science, BIT, Mesra, Ranchi-835215, India \\ 1amaresh.dcs@gmail.com; ${ }^{2}$ uddgam.singh4386@gmail.com; ${ }^{3}$ kksenapati@bitmesra.ac.in
}

\begin{abstract}
The field of computer science is capable of calculating approximate weight of an object through its image. It is new and challenging. The idea behind the weight of an object is nothing but approximation of the pixel carries some weight value which is specific for the corresponding specific object. It is a challenge for the computer science people to develop methods through pixel weight. The main focus of this paper is to find the approximate weight of an object through its digital image. Generally weight calculating machine is used to find out the weight of any object(s). To improve the process and to make it somehow digitized we have proposed a novel method that does not require any physical standard to calculate weight.

Initially, a surface image is captured by the system and whenever a newly taken image, i.e., the image of object placed over the same surface is input, it will be subtracted from our pre-existing surface image. Based on the features of Colour attribute the weight of the object is estimated. From the experimental results, it is observed that the proposed model works perfectly on both training data as well as novel data. We believe this paper could form a base for future works on related topics.

Index Terms - Algorithms, Approximation methods, Colour, Surface image, Weight, Weight per pixel, Weight approximation, Image subtraction, Threshold.

\section{Introduction}

In today's scenario people are becoming more application oriented rather than using physical equipment. In this context, here our work is an approach to meet the goal to some extent which will find the approximate weight of an object from its image in a convincing way.

A digital image is a numeric representation (normally binary) of a two-dimensional image. Depending on whether the image resolution is fixed, it may be of vector or raster type. In a simple way, the term "digital image" usually refers to raster images also called bitmap images. As image consists of millions of pixels, Yao and Odobez described each pixel mode as a combination of intensity and texture [1]. Image features which can be used for retrieval purposes can be in the form of Colour, shape, and texture, as described in [2].Image retrieval by Colour feature is the most widely used method [2]. Our proposed model also utilizes the Colour feature towards the approximation of weight.
\end{abstract}


A background generation and background subtraction approach to separate the moving objects from other components in the image is derived by Yufang Zhang, Peijun Shi, Elizabeth G. Jones, and Qiuming Zhu [3]. The term "Surface image" in our proposed method is used to indicate a background image which has captured before the entrance of any moving object. Ruihua MA, Liyuan LI, Weimin HUANG, Qi TIAN proposed a model where they derived the relation for geometric correction for the ground plane and proves formally that it can be directly applied to all the foreground pixels to estimate the crowd density by pixel counting. They explained there how to implement pixel counting with geometric correction efficiently [4]. Saad Choudri, James M. Ferryman, Atta Badii, in their proposed model, tried to increase the robustness of "Pixel-based people counting" methods when background pixels become increasingly difficult to resolve and to reduce the loss of people when they got absorbed into the background after being stationary for a long period by introducing a selective background update method[5]. In the proposed method also surface image is updated to avoid error reading during pixel counting.

Weight is directly proportionate to the number and type of atoms in the object and physically, atom is the smallest unit in an object, likewise, pixel is the smallest unit for that object in an image and to find the weight of object within the image we have to find the number of pixels covering only for the required object within the image and then finding out the weight per unit pixel or weight per pixel $(w)$. Telling in other way, ' $w$ ' uses the concept of density in spite of the fact that we have kept the word 'density' silent in our paper. The density of a substance is its mass per unit volume and since density varies from substance to substance, same is the case for "weight per pixel" (from object to object). Section-II contains proposed method where the whole process of finding weight per pixel for a specific type of object is derived. After finding out weight per pixel we can accumulate the weight for whole object. Section III derives about the flow of processes, section IV and V contains experimental design and conclusions respectively. Finally section VI contains the references.

\section{Proposed Method}

The proposed method is based on known and similar type of objects. Similar type of objects refer to the objects made up of same materials, e.g., all fishes of the category Paralichthys olivaceus are similar, which in turn are different from fishes of category Oplegnathus fasciatus or any other, wooden pencils come under one category which are different from pencils having plastic sheath, eggs of pigeons are different from eggs of parrots etc.

This method is basically performed in two phases.

\section{1: Pre-processing phase, 2: Execution phase}

In the pre-processing phase some similar type of objects are collected and their weights have been measured manually. Consider the physical weights $W 1, W 2, W 3, W 4, \ldots \ldots . . ., W i n$ respectively are for objects $\mathrm{O} 1, \mathrm{O} 2, \mathrm{O}, \mathrm{O} 4, \ldots, \mathrm{On}$. The average physical weight 'WAvg' is calculated as given below:

$$
\mathrm{W}_{\text {Avg }}=\underset{\mathrm{i}=1}{\left(\sum \mathrm{W}_{\mathrm{i}}\right) / \mathrm{n}}
$$

In the subsequent step the corresponding pixels of the object in the image is counted. Similarly this process is repeated for rest of the images. Now calculate average number of pixels per object. Consider the total number of pixels covering for object $\mathrm{O}_{1}$ is $\operatorname{Pix}\left(\mathrm{O}_{1}\right)$, for object $\mathrm{O}_{2}$, it is Pix $\left(\mathrm{O}_{2}\right)$ and so on. 
Suppose "Pix Avg " is the average number of pixels per object. It can be calculated as:

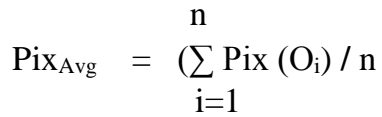

In the next step, the weight per pixel, suppose " $w$ " is calculated as follows:

$$
\begin{aligned}
& w=W_{\text {Avg }} / \text { Pix Avg }_{\text {Ang }} \\
& \mathrm{n} \quad \mathrm{n} \\
& =\left(\left(\sum W_{i}\right) / n\right) /\left(\left(\sum \operatorname{Pix}\left(O_{i}\right)\right) / n\right) \\
& i=1 \quad i=1 \\
& \text { n } \mathrm{n} \\
& =\left(\sum \mathrm{W}_{\mathrm{i}}\right) /\left(\sum \operatorname{Pix}\left(\mathrm{O}_{\mathrm{i}}\right)\right) \\
& \mathrm{i}=1 \quad \mathrm{i}=1 \\
& \mathrm{n} \\
& =\sum\left(\mathrm{W}_{\mathrm{i}} / \operatorname{Pix}\left(\mathrm{O}_{i}\right)\right) \\
& \mathrm{i}=1
\end{aligned}
$$

Now store the value of " $w$ " for further use.

In the execution phase the weight of the required object in a novel image can be finding out in two steps:

Step-1: Find out the number of pixels (Count) covering the object (in novel image) which is to be weighted.

Step-2: The weight of object will be calculated by the following formula as:

$$
\text { Weight of the object }(W)=\text { Count } * w \text {. }
$$

So finally "W" is our desired result. 


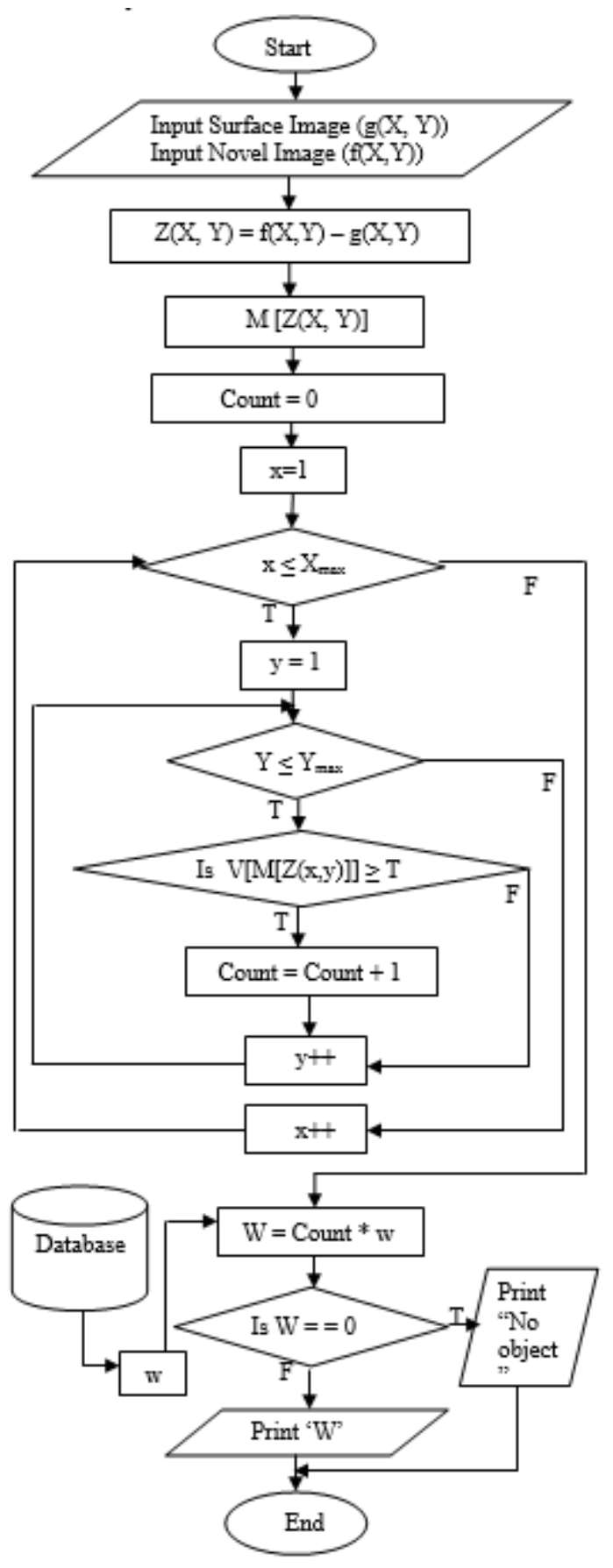

Figure 1: (Flow chart for the proposed method)

\section{Flow of Processes}

As mentioned in the previous section the general principle of the techniques presented in this paper is based on the similar image database for the object.

The activities done are shown in figure-1 briefly which indicates that the input novel image is subtracted immediately from the surface image stored just before the novel image where the object will be placed. Let $f(X, Y)$ be the image to be input and $g(X, Y)$ is the surface image. Both $f(X, Y)$ and $g(X, Y)$ are 2-D images. $Z(X, Y)$ is the resultant image after subtraction of $g(X, Y)$ from $f(X, Y)$. $M[Z(X, Y)]$ is the matrix form of $Z(X, Y)$. Conversions of the resultant image into matrix form simplify the job of separation of 
Amaresh Chandra Meher, Uddgam Singh and K.K. Senapati; A Novel Method for Calculating Approximate Weight of an Object through its Image. Transactions on Machine Learning and Artificial Intelligence, Volume 2 No 6 Dec, (2014); pp: 32-39

object from the background. $X_{\max }$ and $Y_{\max }$ represent the last row and column of the matrix respectively. $\mathrm{V}[\mathrm{M}[\mathrm{Z}(\mathrm{x}, \mathrm{y})]]$ is the value of each pixel in the matrix. The matrix shows that the values of pixels for background in the resultant image (excluding for the object) are zero or nearly equal to zero (due to slight changes in intensity of light) irrespective of the colours of the background. Taking this matter into consideration a threshold value ' $T$ ' for pixels is set. Then all the pixels having value greater than or equal to threshold are counted. These pixels cover for the required object and the rest of the pixels having values below threshold are for background. Initially the counter is set to 0 and is incremented each time for the pixels having values greater than or equal to ' $T$ '. The database already contains ' $w$ ' whose values are objects specific. The appropriate value of ' $w$ ' corresponding to the type of object is found out carefully from the database. Finally to get the approximate weight ' $W$ ' of the object, value of Count is multiplied with the value of ' $w$ '.

\section{Experimental Design and Results}

The equipments required for the experiment are a good quality camera, conveyor belt, the objects to be tested and a processor.

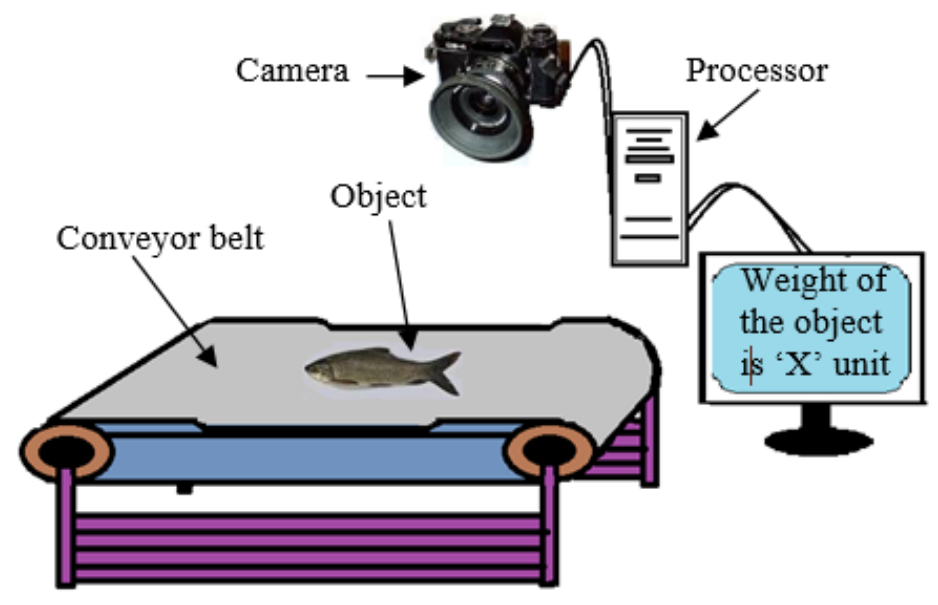

Figure 2

To test the accuracy of the proposed method, thousands of experiments have been performed. Here we have shown the calculated weight of twelve pencils and five no. of "Labeo rohita" fishes by keeping them at the distances $150 \mathrm{~cm}$ and $60 \mathrm{~cm}$ respectively from the camera. The Image of the object is captured only when the object is in maximum contact to the surface. The results produced by our system are compared with the weight calculated by weighting machine.

The proposed method was experimentally verified using a Nikon camera (model no. D3000) keeping resolutions 1936 × 1296 for pencils and images of fishes are taken by a Canon camera (model no. DIGITAL IXUS 95 IS) keeping resolutions $3648 \times 2736$. Horizontal resolution and vertical resolution are $300 \mathrm{dpi}$ for pencils and $180 \mathrm{dpi}$ for fishes. Bit depth is 24 and Colour representation is RBG for both.

For the proposed method the position of the camera is static and its resolutions are kept constant. The colours of surface image and object should not match, as well as the distance between the camera and the surface of the conveyor belt is fixed. The conveyor belt used here has an important characteristic, that is, it always keeps the object placed on it at the proper place to be imaged. In the experiment of 
discussion we used Classmate Carbon Black Pencil. Also for the purpose of clarity in understanding we have provided a surface image, an object image and the resultant image (after subtraction) for a fish whose experimental discussion is not shown here to avoid the lengthiness.

The physical weight of the pencil is $4.6274 \mathrm{gm}$. The surface image and image of pencil are shown in Figure 3 and 4 respectively. The resulted figure after subtraction is shown in Figure 5.

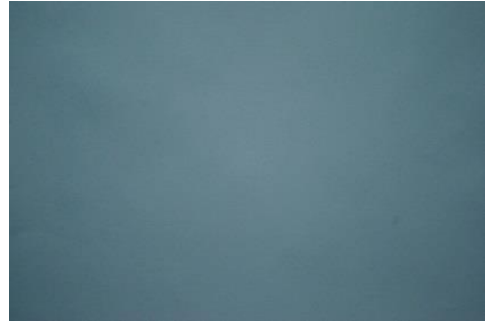

Figure 3

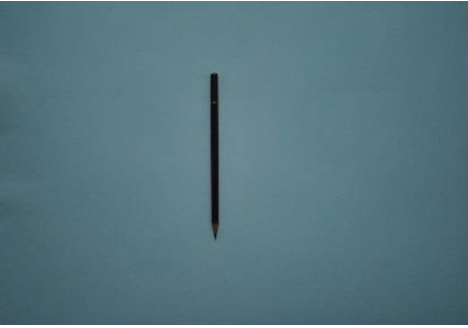

Figure 4

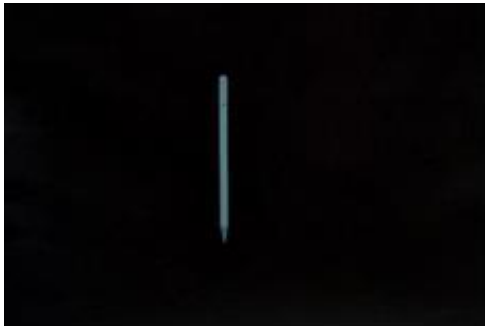

Figure 5

From figure 5, number of pixels (Count) above threshold is 13555 keeping threshold value at 30 . As derived in our proposed method, approximate weight of Classmate carbon Black pencil is the product of Count and ' $w$ '. The value of ' $w$ ' is obtained as $0.0003877529824 \mathrm{gm}$. So the approximate weight of the pencil=13555*0.0003877529824=5.255992gm.

Table 1

\begin{tabular}{|c|c|c|}
\hline Pencil Name & $\begin{array}{c}\text { Physical } \\
\text { Weight }\end{array}$ & $\begin{array}{c}\text { Number of pixels } \\
\text { covering the pencil }\end{array}$ \\
\hline $\begin{array}{c}\text { Ole-Grip } \\
\text { (super dark) }\end{array}$ & 5.17710 & 13167 \\
\hline Camlin Flora & 4.7574 & 14693 \\
\hline $\begin{array}{c}\text { Nataraj } 621 \\
\text { (HB) }\end{array}$ & 4.6552 & 13633 \\
\hline $\begin{array}{c}\text { Papyrus } \\
\text { (HB) }\end{array}$ & 5.9298 & 12678 \\
\hline POLO CHECKING & 6.3607 & 15152 \\
\hline
\end{tabular}

Taking into consideration for five pencils as shown in table-1, value of ' $w$ ' can be calculated where values of $W_{\text {avg }}$ and Pixavg are calculated as 5.37604 and 13864.6 respectively.

Weight per pixel ' $w$ ' $=\left(W_{\text {Avg }} /\right.$ Pix $\left.x_{\text {Avg }}\right)$

Physical weight of classmate carbon pencil is $4.6274 \mathrm{gm}$ and with our proposed method it is coming as $5.255992 \mathrm{gm}$. So the accuracy is of $88.04 \%$.

Similarly many experiments have been performed through the proposed method whose results are presented in table -2 \& table- 3 with its accuracy. Table- 2 is for pencils and table- 3 is for fishes. 
Table 2

\begin{tabular}{|c|c|c|c|}
\hline Name of pencils & $\begin{array}{c}\text { Weight } \\
\text { calculated } \\
\text { by } \\
\text { Weighting } \\
\text { Machine } \\
\text { (in gm) }\end{array}$ & $\begin{array}{c}\text { Results } \\
\text { produced by } \\
\text { our system } \\
\text { (in gm) }\end{array}$ & Accuracy \\
\hline Ole-Grip: (super dark) & 5.17710 & 5.80389 & 89.20 \\
\hline Papyrus: (HB) & 5.9298 & 4.915932 & 82.90 \\
\hline Camlin Flora & 4.7574 & 4.00450 & 84.17 \\
\hline Nataraj 621: (HB) & 4.6552 & 5.286236 & 86.44 \\
\hline Flassmate:(Carbon black) & 4.6274 & 5.25599 & 88.04 \\
\hline RUN (ULTRA DARK BONDED LEAD) & 5.0082 & 5.765111 & 86.87 \\
\hline REYNOLDS HB & 4.8439 & 5.811642 & 83.35 \\
\hline POllo LEADER HB & 4.9393 & 5.502603 & 89.76 \\
\hline APSARA Platinum (Extra Dark) & 4.5673 & 5.097013 & 89.61 \\
\hline APSARA DRAWING 701 & 4.8490 & 5.874458 & 82.54 \\
\hline STOR HB & 3.6607 & 3.734061 & 98.04 \\
\hline
\end{tabular}

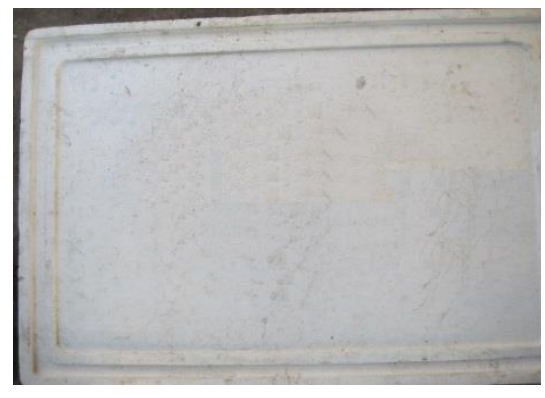

Figure 6: (Surface image for the fish)

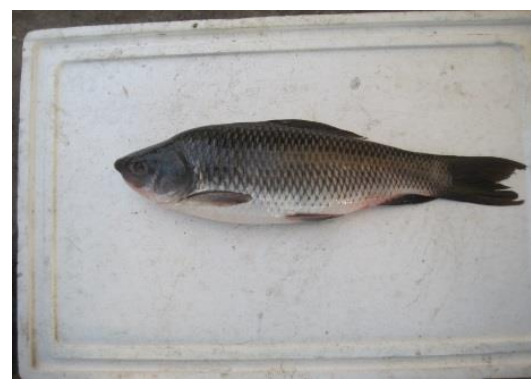

Figure 7:(Object image for the fish)

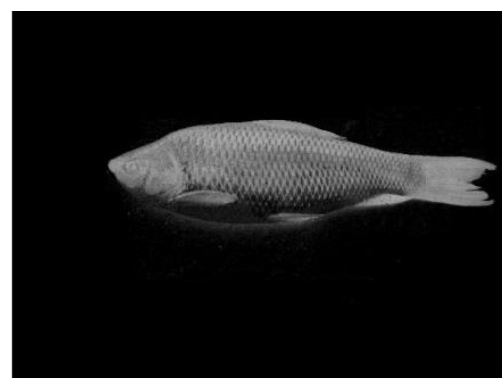

Figure 8: (Resultant image)

Table 3

\begin{tabular}{|c|c|c|c|}
\hline $\begin{array}{c}\text { Weight } \\
\text { calculated } \\
\text { by } \\
\text { Weighting } \\
\text { Machine } \\
\text { (in gm) }\end{array}$ & $\begin{array}{c}\text { Results } \\
\text { produced by } \\
\text { our system } \\
\text { (in gm) }\end{array}$ & $\begin{array}{c}\text { Number of } \\
\text { pixels } \\
\text { covering } \\
\text { object }\end{array}$ & Accuracy \\
\hline 1000 & 9979.51988 & 1074969 & 99.98 \\
\hline 1100 & 1185.836874 & 1274999 & 92.83 \\
\hline 1200 & 1256.285024 & 1350744 & 95.52 \\
\hline 1400 & 1341.91173 & 1442809 & 95.85 \\
\hline 1450 & 1472.456197 & 1583169 & 98.51 \\
\hline \multicolumn{3}{|c|}{ Threshold = 34, w = 0.000930068866 } \\
\hline
\end{tabular}

\section{Conclusion}

In this paper we have presented a novel method for finding the weight of an object(s) through an image and the result of the method as observed is nearly equals to the actual weight of the object which shows that our method works properly. Again more precise result can be obtained by further prudent work on this. 
As author we must accept that this paper contains lot of constraints and it works only for a limited category of objects in the real world but keeping in mind that as this is the first step towards the goal constraints must be there. We also feel strongly that the idea is new and unique.

This method can also be useful in photographic industry, study of celestial objects and in many more areas.

This is an initial attempt; further enhancement on this technique can facilitate capturing images by a portable device. This novel technique can be further upgraded for dissimilar object in a single frame with the aid of object recognition.

\section{REFERENCES}

[1] J.Yao and J.Odobez, "Multi-layer background subtraction based on color and texture," IEEE Conference on Computer Vision and Pattern Recognition, pp.1-8, 2007.

[2] A.K. Jain, "Fundamentals of Digital Image Processing", Englewood cliffs, NJ: Prentice-Hall, 1980.

[3] Yufang Zhang, Peijun Shi, Elizabeth G. Jones, and Qiuming Zhu" Robust Background Image Generation and Vehicle 3D Detection and Tracking". 2004 IEEE Intelligent Transpoltation Systems Conference Washington, D.C., USA, October 36, 2004.

[4] Ruihua MA, Liyuan LI, Weimin HUANG, QiTIAN, "On Pixel Count Based Crowd DensityEstimation for Visual Surveillance" Proceedings of the 2004 IEEE Conference on Cybernetics and Intelligent Systems Singapore, 1-3 December,2004

[5] Saad Choudri, James M. Ferryman, Atta Badii, "Robust Background Model for Pixel Based People Counting using a Single Uncalibrated Camera", 978-1-4244-5504-1/09/\$25.00 @2009 IEEE 